\title{
The ecology of infants' perceptual-motor exploration
}

\author{
John M. Franchak \\ Department of Psychology, University of California, Riverside \\ 900 University Avenue, Riverside, CA
}

\begin{abstract}
The first goal of this article is to review recent advances in understanding how new motor skills facilitate infants' exploration - the active acquisition of information about their environments. New postural abilities, such as sitting and walking, qualitatively change how infants can learn about objects, places, and people with with potential downstream effects on infants' later cognitive and linguistic development. What's missing, however, is a characterization of how new exploratory abilities change infants' everyday experiences. Presumably, changes in opportunities for learning mediate the downstream effects of posture on other developmental achievements. Accordingly, the second goal of this article is to discuss the importance of measuring the ecology of infants' everyday experiences and how they vary.
\end{abstract}

Keywords: Ecological psychology, exploration, perceptual-motor development, posture

\section{Introduction}

Ecological and systems theories emphasize the interdependence between individual and environment [1-[5]. For example, James Gibson's concept of affordances considers how an individual's motor capabilities with respect to physical characteristics of the environment co-determine whether actions are possible [2]. The Gibsonian concept of exploration - how observers' motor actions support

Email address: franchak@ucr.edu (John M. Franchak)

Preprint submitted to Current Opinion in Psychology

June 26, 2019 
actively acquiring information about the environment - also reflects individualenvironment interdependence [6, 7]. Eleanor Gibson extended this idea to infant development, wherein the acquisition of new motor abilities fundamentally changes how infants can explore - and thus learn about-their environments [3. Gibson's claim has been borne out: The acquisition of motor skills, such as sitting, crawling, and walking, predicts developmental changes in perception, cognition, and language [e.g., $8-10$.

The first goal of this article is to review recent work documenting how motor development - more specifically, the acquisition of new postures - augments exploration and effects broader changes on cognitive and language development. However, I will argue that despite a solid foundational understanding in how exploratory abilities change, we know relatively little about how exploratory experiences change. The degree to which exploratory abilities influence later development-so-called downstream effects - are presumably mediated by the everyday exercise of exploration. Just as affordances are co-determined by the individual-environment relation, I contend that infants' everyday opportunities for learning are co-determined by their exploratory abilities relative to what their environments offer. Environments vary between infants (e.g., cultural and material factors) and over time (e.g., changes in caregiving). Thus, the second goal of this article is to discuss the importance of characterizing everyday exploration, identify the challenges of studying those experiences, and review encouraging steps taken to address this gap.

\section{Postural development alters exploration}

Without the ability to control posture, young infants can only lie supine on their backs or prone on their stomachs unless they are held by caregivers, and they must rely on caregivers to switch between different positions. Over the first two years, infants master static postures (sitting and standing) and dynamic postures (crawling and walking) that dramatically expand exploratory abilities [1]. 
Infants learn about objects through coordinated visual, manual, and oral exploration. Although the ability to grasp and acquire objects spurs object exploration [12, further developments depend on infants' ability to sit. Coordinating the hands to rotate, transfer, and manipulate objects - especially while simultaneously looking at objects - is facilitated by sitting compared to prone and supine postures [9, 13]. Using the hands to support the body when prone hinders coordinated visual-manual exploration. Sitting frees both hands to manipulate objects, and the stability of sitting allows infants to better control their heads to look at objects [9, 14].

Posture not only influences exploration of near objects, but also constrains visual exploration of distant targets. Recent advances in mobile eye tracking technology have allowed researchers to measure where infants look in naturalistic settings [15-17]. Whereas more commonly-used methods, such as measuring looking towards stimuli on a screen, preclude studying visual attention as an embodied process, mobile eye tracking captures how every turn of the head and body alter what infants see (Figure 1). Results reveal that when sitting and upright, 13-month-oldw see farther in the distance compared to when prone [18. Prone infants' view is dominated by the floor, as they must struggle to lift up their heads to glimpse distant locations. This impacts how often infants look at important visual targets. For example, posture influences social looking (Figure 2): 12-month-olds rarely look at caregivers' faces when prone compared with sitting and upright postures [19]. However, face looking is still quite rare ( $\sim 5 \%$ of the time) when sitting and upright, in part because caregivers' faces are too high up to be seen by infants playing on the floor. Instead, infants look at caregivers' bodies ( $\sim 15 \%$ of the time) - their hands, legs, and torsos - which are easier to view from infants' low vantage point. A higher vantage point (such as when carried) allows infants to more frequently look at faces $(\sim 18 \%$ of the time) [20].

Acquiring locomotor abilities - crawling and walking - provides new ways to explore. Before crawling, infants must rely on caregivers to move them from place to place: A sitting infant may glimpse a distant toy, but a crawling infant 

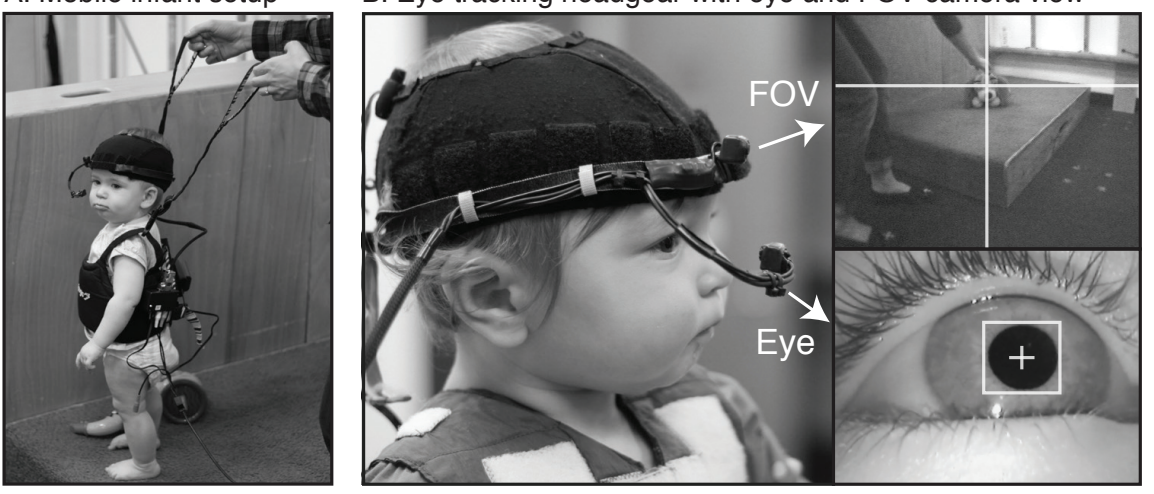

Figure 1: Mobile eye-tracking method. A) Infant wearing mobile eye tracker in a laboratory playroom. B) Close-up of infant eye-tracking headgear, which houses two cameras to capture the infant's eye and the approximate field of view (FOV). Calibration of eye and field of view videos allows tracking of gaze position within the field of view, indicated by a crosshair.

can go retrieve it. With greater crawling experience, infants gain autonomy and travel farther from their caregivers. The acquisition of walking provides even more advantages over crawling: Walking entails greater speed of movement [21, 22], allows infants to travel greater distances [21, 23], facilitates carrying objects [24, 25, and provides a better viewpoint of distant locations [18. Both crawling and walking alter social interactions with caregivers [8, 23, 26]. For example, walking compared with crawling allows infants to bring distant objects

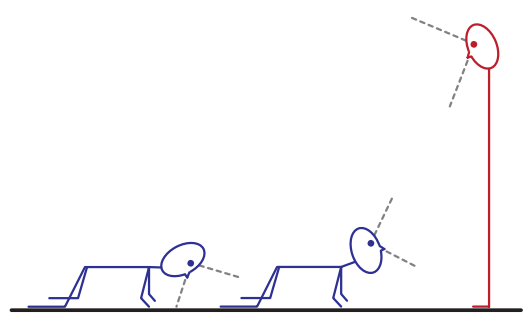

Prone/crawling

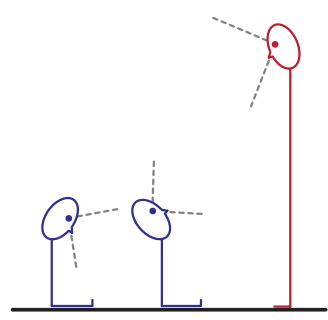

Sitting

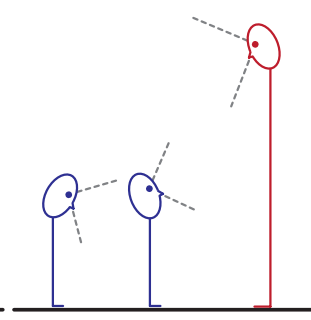

Standing/walking

Figure 2: Infant viewpoint of a standing caregiver from prone, sitting, and upright postures. Infant head angles are drawn to approximate the ranges reported in 18 . 
to share with caregivers [24].

\section{Changes in exploratory abilities have downstream effects}

The varied ways by which posture alters exploration suggests multiple pathways through which posture may influence other areas of development. For brevity, I will review how postural attainment facilitates later spatial and linguistic abilities. However, it is important to consider that acquiring new motor skills may not always be facilitative, especially in the short term. Crawling interferes with new crawlers' vocalizations [27] and carrying objects disrupts new walkers' balance [28].

The attainment of sitting, crawling, and walking have downstream effects on object perception [9] and spatial cognition [8, 29]. Infants' ability to sit independently facilitates visual-manual exploration of objects [13, 14], which, in turn, facilitates object perception [9. Downstream effects can be far-reaching: Earlier sitting onsets predict better spatial memory at 24 months [29]. Although object exploration at 20 months failed to mediate this effect, exploratory differences earlier in infancy might account for it. Independent crawling and walking predict earlier achievement in spatial cognition tasks [8, 29]. Infants who walked earlier engaged in more exploratory locomotion at 20 months, which predicted better performance on a standardized block-copying task at 32 months [29]. Other exploratory benefits of walking over crawling, such as a better viewpoint for distant locations [18] and the ability to carry objects [24, 25], have yet to be tested as potential mediators in downstream effects on spatial abilities.

Earlier attainment of both sitting [29, 30] and walking [29, 31, 32] predict receptive and productive vocabulary between 1 and 4 years. Unlike spatialcognitive abilities, downstream effects of sitting on language were not mediated by object exploration [29, 30. Other, untested aspects of object exploration may be more relevant. Recent work indicates that infants' self-generated visual experiences (greater variability of object views when holding and moving objects) predict vocabulary growth [33], but whether sitting ability facilitates 
variability in object experiences is unknown. Furthermore, sitting facilitates shared visual attention to objects between infants and caregivers [19] and might enhance opportunities for language learning. Similarly, joint attention partially mediates the effect between walking onset and vocabulary development [31]. Most likely, sitting and walking have cascading effects on later language development through multiple pathways, such as changes in caregivers' behaviors in response to infants' motor development. Caregivers provide more complex language input to 13-month-old walkers compared with crawlers [34]. Moreover, caregivers' perception of infants as individuals increases after infants begin to walk, which predicts later vocabulary development [31].

\section{What's missing: The ecology of everyday exploratory experiences}

A key assumption in linking exploratory abilities to downstream effects is that they are mediated by changes in everyday experiences. But direct measurements of daily experiences are rare [35], and inferences based on laboratory observations might not generalize well to daily life. Brief laboratory play tasks cannot capture infants' full daily routines, which contain both play and non-play activities (e.g., feeding, bathing, errands) that may provide different opportunities for exploration. For example, in a mobile play task infants may be unrestrained [19, 23], but in everyday feeding and travel activities infants are restrained in high chairs, strollers, and car seats. Infants' and caregivers' behaviors may also be biased by researchers' observation. For example, caregivers spoke up to four times more during audio-video recordings compared with audio-only recordings [36].

Recent advances in wearable devices - lightweight "headcams" that record infant-perspective visual experiences and audio recorders that can capture daylong recordings of infant and adult speech - provide researchers with new ways to capture home experiences at scale [36-38]. Headcam recordings over the first two years of life show that the frequency of faces in infants' views decreases with age while that the presence of hands increases in frequency [37. The differential 
quality and frequency of visual experiences of objects in the home may shed light on why and how infants learn specific words [39, 40].

Given the hypothesized downstream effects of sitting, crawling, and walking, it will be important in future work to characterize everyday motor experiences throughout the day. But whereas infants' language and visual experiences can be profitably measured with audio recorders and headcams, the gold standard for measuring infants' naturalistic motor activity is third-person video in the home [41] or laboratory [19, 23, which suffers from the limitations mentioned above. One alternative is to use ecological momentary assessment (EMA). Changes in infants' posture over the first year of life 42] were captured through caregiver reports in response to 35 text message notifications over a week. These full-day recordings differed from brief laboratory observations: 12-month-olds were upright $50-70 \%$ of the time in laboratory play [19, 23] compared with $30 \%$ of the time in the home. A future alternative may be to use lightweight inertial sensors [38: Posture has been accurately classified in small samples of infants in controlled tasks [43], but soon these methods may be suitable for home recordings.

Several open questions remain. First, how does the frequency of exploratory experiences change after acquiring a new exploratory ability? For example, both EMA [42] and video [41] recordings indicate that 5- to 6-month-old independent sitters sit (with or without support from caregivers or furniture) twice as often as same-aged non-sitters. More sitting time could increase opportunities for object exploration or shared attention with caregivers, but these opportunities - and whether they mediate downstream effects on cognition and language-have yet to be tested. Similarly, postural effects on visual exploration in the lab have yet to be extended to measurement of everyday visual experiences - simultaneous headcam and EMA/inertial measurement could address this question and potentially contribute to our understanding of word learning [e.g., 33].

Second, what does the environment offer for exploration - how do environments vary and how do they change? Future work should characterize the everyday motor opportunities afforded by infants' environments, how they vary, and what factors (e.g., caregiving, material, and/or cultural) account for vari- 
ations. For example, use of infant equipment to restrain infants is common in the US, and individual differences in use depend on factors such as family size [44. But cross-cultural studies show that infants' experiences with motor restraint can exceed what is typical for US infants. In Tajikistan, 12-month-olds spend between as many as 18.5 hours each day restrained on their backs [45, 46]. Future research should determine whether individual differences in restraint (depending on caregiving and cultural factors) constrain infants' opportunities to explore, with potential downstream effects on cognition and language.

A second source of variation is the home environment. Infants' access to material resources relevant for perceptual-motor exploration vary widely by socioeconomic and cultural factors [47]. The Affordances in the Home Environment for Motor Development scale (AHEMD) characterizes variability in the physical environment related to motor development and predict standard measures of motor skill acquisition [48]. In particular, the number of objects for promoting fine and gross motor skills and the total amount of space in the home are linked with motor development [49, 50]. More work, however, is needed to determine whether perceptual-motor exploration is also facilitated by these variations in the physical environment.

\section{Conclusion}

There is little doubt from the prevailing literature that changing motor abilities alter opportunities for learning. But the extent to which infants actualize those opportunities in daily life is largely unknown, particularly with regard to postural activities and their potential consequences on visual, manual, and social experiences. New recording methods show promise for describing the ecology of infants' everyday experiences and how they are shaped by environmental variation. 


\section{References}

[1] Bronfenbrenner U. Toward an experimental ecology of human development. American Psychologist 1977;32:513-31.

[2] Gibson JJ. The ecological approach to visual perception. Boston, MA: Houghton Mifflin Company; 1979.

[3] Gibson EJ. Exploratory behavior in the development of perceiving, acting, and the acquiring of knowledge. Annual Review of Psychology 1988;39:141.

[4] Thelen E, Smith LB. A dynamic systems approach to the development of cognition and action. Cambridge, MA: MIT Press; 1994.

[5] Oishi S, Graham J. Social ecology: Lost and found in psychological science. Perspectives on Psychological Science 2010;5:356-77.

[6] Gibson JJ. The senses considered as perceptual systems. Boston: Houghton-Mifflin; 1966.

[7] Franchak JM. Looking with the eyes and head. In: Wagman JB, Blau JJC, editors. Perception as information detection: Reflections on Gibson's Ecological Approach to Visual Perception. Routledge; 2020,.

[8] Campos JJ, Anderson DI, Barbu-Roth MA, Hubbard EM, Hertenstein MJ, Witherington DC. Travel broadens the mind. Infancy 2000;1:149-219.

[9] Soska KC, Adolph KE, Johnson SP. Systems in development: Motor skill acquisition facilitates three-dimensional object completion. Developmental Psychology 2010;46:129-38. doi:10.1037/a0014618.

[10] Libertus K, Hauf P. Motor skills and their foundational role for perceptual, social, and cognitive development. Frontiers in Psychology; 2017. **This is an edited collection of empirical studies that document various downstream effects of changes in motor skills on later development. 
[11] Adolph KE, Franchak JM. The development of motor behavior. WIREs Cognitive Science 2017;8:e1430.

[12] Needham AW, Wiesen SE, Hejazi JN, Libertus K, Christopher C. Characteristics of brief sticky mittens training that lead to increases in object exploration. Journal of Experimental Child Psychology 2017;164:209-24.

[13] Soska KC, Adolph KE. Postural position constrains multimodal object exploration in infants. Infancy 2014;19:138-61. doi:10.1111/infa.12039.

[14] Marcinowski EC, Tripathi T, Hsu LY, Westcott McCoy S, Dusing SC. Sitting skill and the emergence of arms-free sitting affects the frequency of object looking and exploration. Developmental Psychobiology in press;.

[15] Franchak JM, Kretch KS, Soska KC, Adolph KE. Head-mounted eye tracking: A new method to describe infant looking. Child Development 2011;82:1738-50. doi:10.1111/j.1467-8624.2011.01670.x.

[16] Franchak JM. Using head-mounted eye tracking to study development. In: Hopkins B, Geangu E, Linkenauger S, editors. The Cambridge Encyclopedia of Child Development; 2nd ed. Cambridge: Cambridge University Press; 2017, p. 113-6.

[17] Slone LK, Abney DH, Borjon JI, Chen Ch, Franchak JM, Pearcy D, et al. Gaze in action: Head-mounted eye tracking of children's dynamic visual attention during naturalistic behavior. Journal of Visualized Experiments 2018;141:e58496.

[18] Kretch KS, Franchak JM, Adolph KE. Crawling and walking infants see the world differently. Child Development 2014; doi:10.1111/cdev.12206.

[19] Franchak JM, Kretch KS, Adolph KE. See and be seen: Infantcaregiver social looking during locomotor free play. Developmental Science 2018;21:e12626. *This study used mobile eye tracking during infantcaregiver play to demonstrate that infants' viewpoint (as determined by posture) affects the frequency of face looking and shared attention. 
[20] Kretch KS, Adolph KE. Active vision in passive locomotion: Real-world free viewing in infants and adults. Developmental Science 2015;18:736-50. doi:10.1111/desc.12251.

[21] Adolph KE, Tamis-LeMonda CS. The costs and benefits of development: The transition from crawling to walking. Child Development Perspectives 2014;8:187-92. doi:10.1111/cdep.12085.

[22] Lee DK, Do Kyeong L, Cole WG, Golenia L, Adolph KE. The cost of simplifying complex developmental phenomena: A new perspective on learning to walk. Developmental Science 2018;21:e12615.

[23] Thurman SL, Corbetta D. Spatial exploration and changes in infant-mother dyads around transitions in infant locomotion. Developmental Psychology 2017;53:1207-21.

[24] Karasik LB, Tamis-LeMonda CS, Adolph KE. Transition from crawling to walking and infants' actions with objects and people. Child Development 2011;82:1199-209. doi $10.1111 / \mathrm{j}$.1467-8624.2011.01595.x.

[25] Karasik LB, Adolph KE, Tamis-LeMonda CS, Zuckerman A. Carry on: Spontaneous object carrying in 13-month-old crawling and walking infants. Developmental Psychology 2012;48:389-97. doi 10.1037/a0026040.

[26] Thurman SL, Corbetta D. Spatial exploration and changes in infant-mother dyads around transitions in infant locomotion. Developmental Psychology in press;

[27] Berger SE, Cunsolo M, Ali M, Iverson JM. The trajectory of concurrent motor and vocal behaviors over the transition to crawling in infancy. Infancy $2017 ;: 681-94$.

[28] Heiman CM, Cole WG, Lee DK, Adolph KE. Object interaction and walking: Integration of old and new skills in infant development. Infancy $2019 ; 66$. 
[29] Oudgenoeg-Paz O, Leseman PPM, Volman MCJM. Exploration as a mediator of the relation between the attainment of motor milestones and the development of spatial cognition and spatial language. Developmental Psychology 2015;51:1241-53.

[30] Libertus K, Violi DA. Sit to talk: Relation between motor skills and language development in infancy. Frontiers in Psychology 2016;7:e475.

[31] Walle EA. Infant social development across the transition from crawling to walking. Frontiers in Psychology 2016;7:e960.

[32] Oudgenoeg-Paz O, Volman MCJM, Leseman PPM. First steps into language? examining the specific longitudinal relations between walking, exploration and linguistic skills. Frontiers in Psychology 2016;7:e1458.

[33] Slone LK, Smith LB, Yu C. Self-generated variability in object images predicts vocabulary growth. Developmental Science 2019;:e12816.

[34] Karasik LB, Tamis-LeMonda CS, Adolph KE. Crawling and walking infants elicit different verbal responses from mothers. Developmental Science 2014;17:388-95. doi:10.1111/desc.12129.

[35] Dahl A. Ecological commitments: Why developmental science needs naturalistic methods. Child Development Perspectives 2017;11:79-84.

[36] Bergelson E, Amatuni A, Dailey S, Koorathota S, Tor S. Day by day, hour by hour: Naturalistic language input to infants. Developmental Science 2019;22:e12715. **The authors used LENA recordings to document infants' naturalistic language input in the home. Comparing periods during which caregivers were or were not video recorded revealed that video recording may bias measurement of naturalistic language input.

[37] Smith LB, Jayaraman S, Clerkin E, Yu C. The developing infant creates a curriculum for statistical learning. Trends in Cognitive Sciences 2018;22:325-36. *The authors review naturalistic and semi-naturalistic 
studies of infants' visual experiences using headcams and eye trackers and discuss how those experiences relate to visual and language learning.

[38] Barbaro K. Automated sensing of daily activity: A new lens into development. Developmental Psychobiology in press;*A recent review of advances in mobile sensing technology for studying infants' daily experiences, including mobile audio recording and inertial tracking.

[39] Clerkin EM, Hart E, Rehg JM, Yu C, Smith LB. Real-world visual statistics and infants' first-learned object names. Philosophical Transactions of the Royal Society B 2017;372:20160055. *The authors used headcam recordings in the home environment to document the frequency of visual experiences of particular household objects, and linked those experiences to early noun learning.

[40] Suanda SH, Barnhart M, Smith LB, Yu C. The signal in the noise: The visual ecology of parents' object naming. Infancy 2018;23:1387.

[41] Karasik LB, Tamis-LeMonda CS, Adolph KE, Bornstein MH. Places and postures: A cross-cultural comparison of sitting in 5-month-olds. Journal of Cross-Cultural Psychology 2015;46:1023-38.

[42] Franchak JM. Changing opportunities for learning in everyday life: Infant body position over the first year. Infancy 2018;24:187-209.

[43] Nam Y, Park JW. Child activity recognition based on cooperative fusion model of a triaxial accelerometer and a barometric pressure sensor. IEEE Journal of Biomedical and Health Informatics 2013;17:420-6.

[44] Fay D, Hall M, Murray M, Saatdjian A, Vohwinkel E. The effect of infant exercise equipment on motor milestone achievement. Pediatric Physical Therapy 2006;18:90.

[45] Karasik LB, Tamis-LeMonda CS, Ossmy O, Adolph KE. The ties that bind: Cradling in tajikistan. PloS One 2018;13:e0204428. **An investigation of 
the practice of cradling in Tajikistan provides an extreme example of how cultural factors may influence infants' opportunities for perceptual-motor exploration.

[46] Karasik LB. Mobility: Crawling and walking. In: Shackelford TK, WeekesShackelford VA, editors. Encyclopedia of Evolutionary Psychological Science. Cham: Springer International Publishing; 2018, p. 1-11.

[47] Bradley RH, Putnick DL. Housing quality and access to material and learning resources within the home environment in developing countries. Child Development 2012;83:76-91.

[48] Caçola P, Gabbard C, Santos DCC, Batistela ACT. Development of the affordances in the home environment for motor Development-Infant scale. Pediatrics International 2011;53:820-5.

[49] Saccani R, Valentini NC, Pereira KRG, Müller AB, Gabbard C. Associations of biological factors and affordances in the home with infant motor development. Pediatrics International 2013;55:197-203.

[50] Mori S, Nakamoto H, Mizuochi H, Ikudome S, Gabbard C. Influence of affordances in the home environment on motor development of young children in japan. Child Development Research 2013;:e898406. 